\title{
Influence of the autonomic nervous system on circadian patterns of myocardial ischaemia: comparison of stable angina with the early postinfarction period
}

\author{
Bradley Marchant, Robert Stevenson, Sudhir Vaishnav, Paul Wilkinson,
} Kulasagaram Ranjadayalan, Adam D Timmis

\begin{abstract}
Objective-To compare the circadian rhythm of myocardial ischaemia in patients with stable angina with that in patients in the early postinfarction period with particular emphasis on the role of the autonomic nervous system.

Patients-44 patients with stable angina and ischaemia on treadmill testing (group A) were compared with 131 patients in the early postinfarction period (group B). All had 48 hour ambulatory Holter monitoring.

Setting-Coronary care unit and cardiology department of a district general hospital.
\end{abstract}

Design-Prospective, between group, comparative study.

Results-337 ischaemic episodes occurred in 35 patients in group $A$ and 370 ischaemic episodes occurred in 65 patients in group B. $34 \%$ of patients in group A had only silent episodes of ischaemia compared with $97 \%$ in group B (p< 0.0001). In group $A$ ischaemic episodes showed a circadian rhythm that peaked during the daytime hours $(p<$ $0 \cdot 0001$ ), but this was not seen in group $B$. Both the high $(0.15-0.40 \mathrm{~Hz})$ and low $(0.04-0.15 \mathrm{~Hz})$ frequency spectral components of heart rate variability showed a clear circadian rhythm $(p<0.0001)$; peak values occurred during the sleeping hours, although this pattern was less pronounced in group $B$. The ratio of low to high frequency variability (a measure of sympathovagal balance) showed a peak in daytime hours in group $A(p<0.002)$, but this was not seen in group $B$.

Conclusion-In stable angina, myocardial ischaemia peaks during the day and is associated with a similar circadian rhythm of sympathovagal balance. In the early postinfarction period both the ischaemic and sympathovagal rhythms are severely diminished or lost altogether. Circadian changes in sympathovagal tone may explain, at least in part, the circadian rhythm of ambulatory myocardial ischaemia in patients with stable angina.

(Br Heart f 1994;71:329-333)

Circadian rhythms are now established in ischaemic heart disease, ${ }^{1-3}$ and examining such patterns may help to understand the underlying pathophysiology. For example, myocardial infarction is more common in the second quarter of the day, ${ }^{45}$ and this may, in part, be due to diurnal fluctuations in platelet ${ }^{6}$ and fibrinolytic factors. ${ }^{78}$ Also, an increase in sympathetic activity is likely to contribute to the increased incidence of acute myocardial infarction in the immediate hours after waking. ${ }^{10}$

Circadian rhythms in patients with stable angina have been extensively studied. ${ }^{11}$ Ischaemia seems to be greatest in the second quarter of the day, with a secondary peak in the late afternoon. ${ }^{1}$ There are, however, few data on such rhythms in the postinfarction period, ${ }^{12}$ when myocardial ischaemia is common and usually silent. ${ }^{12-14}$ Such ischaemia may indicate a poor prognosis ${ }^{13}$ and the mechanism causing it is therefore of interest. Autonomic function is deranged in the postinfarction period, ${ }^{15}$ and may play a part in the pathophysiology of ischaemia in these patients.

This study has therefore been designed to compared the circadian rhythms of myocardial ischaemia in patients with stable angina with patients in the postinfarction period, putting particular emphasis on the role of the autonomic nervous system.

\section{Methods}

PATIENTS WITH STABLE ANGINA (GROUP A)

Consecutive patients were recruited from those undergoing exercise treadmill tests for the assessment of angina. All patients were required to have a normal resting 12 lead electrocardiogram to minimise ambiguity in interpretation of ST changes during exercise and ambulatory monitoring. Forty four patients fulfilled these criteria and developed ischaemia on exercise treadmill tests (defined as $\geqslant 0.1 \mathrm{mV}$ of planar or down sloping ST segment depression) and 41 underwent coronary angiography to confirm significant coronary artery disease (defined as at least one stenosis of $\geqslant 75 \%$ in one or more of the main coronary arteries). Antianginal medication was withdrawn five days before the study with the exception of short acting nitrates, which were disallowed only during the study.

PATIENTS AFTER ACUTE MYOCARDIAL INFARCTION (GROUP B)

Patients were recruited from those treated with thrombolysis for acute myocardial
St Bartholomew's Hospital, London EC1A 7BE.

Accepted for publication 27 October 1993 
infarction. Myocardial infarction was confirmed if at least two of the following criteria were fulfilled; (a) typical chest pain lasting $>30$ minutes; ( $b$ electrocardiographic changes of $Q$ wave or non- $Q$ wave myocardial infarction; (c) rise in serum creatine kinase to $>400$ IU/1. Only patients with confirmed myocardial infarction and a stable course in hospital who were not taking $\beta$ blocking drugs were considered for inclusion. Patients with repolarisation abnormalities caused by left bundle branch block, paced rhythms, and concurrent digoxin treatment were excluded. One hundred and thirty one patients fulfilled these criteria and underwent Holter monitoring.

\section{AMBULATORY HOLTER MONITORING}

All patients underwent 48 hour ambulatory Holter monitoring of ST segments. Recordings were made in group $\mathrm{B}$ at 88 (range $48-235$ ) hours after the onset of infarction when the patients were mobile. Lead CMV5 and modified lead II were used, and recordings were made with a Marquette Series 8000 recorder. These were analysed
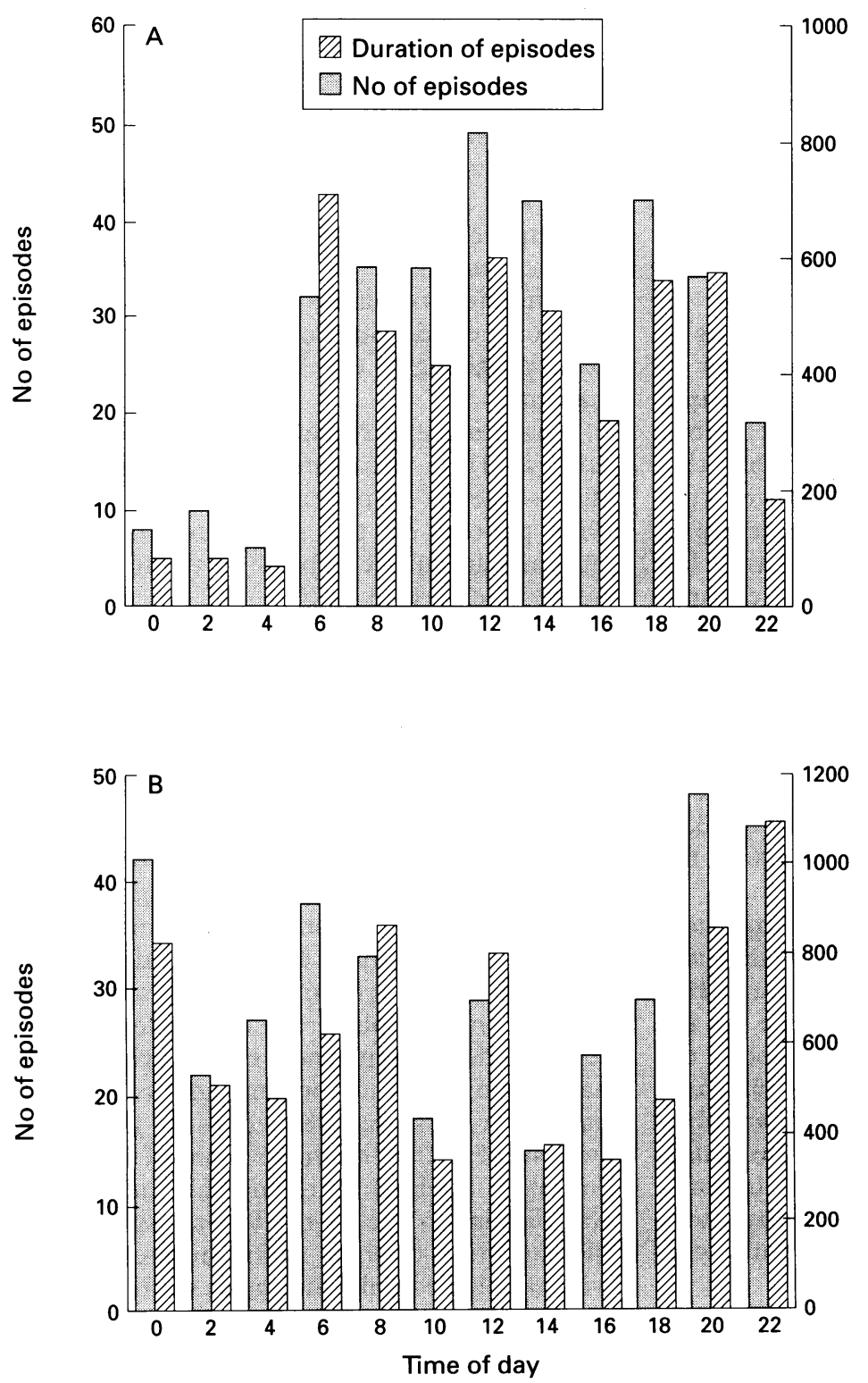

Figure 1 Circadian variation in the number and duration of ischaemic episodes over 12 two hour periods in patients with $(A)$ stable angina (group $A)$, and $(B)$ after myocardial infarction (group B). with a Marquette Holter Acquisition Module with software version $5 \cdot 8$. The time and duration of episodes of ST depression were documented. An episode was defined as $\geqslant$ $0 \cdot 1 \mathrm{mV}$ of planar or down sloping ST segment depression lasting for $\geqslant 1 \mathrm{~min}$; the $\mathrm{ST}$ segment was required to return to baseline for two minutes before a second episode could be counted. The occurrence of anginal symptoms was indicated by pressing a button on the recorder and keeping a dairy.

\section{HEART RATE VARIABILITY}

The first 24 hours of Holter recording were analysed for spectral and non-spectral measures of heart rate variability with Marquette heart rate variability software. The measures calculated were: amplitude of low $(0.04-0.15 \mathrm{~Hz})$ and high $(0.15-0.40 \mathrm{~Hz})$ frequency spectral analysis for each hour, proportion of adjacent RR intervals more than $50 \mathrm{~ms}$ different ( $\mathrm{pNN} 50$ ), root mean square of difference of successive $R R$ intervals (rMSSD), mean of all 5 min standard deviations of RR intervals (SD), standard deviation of 5 min mean RR intervals (SDANN), and the standard deviation of all $R R$ intervals (SDRR). Only patients with adequate quality recordings for the whole 24 hours were included in analysis of heart rate variability. There were 24 such patients in group A and 36 in group B. Low frequency to high frequency amplitude ratios were calculated and plotted for hourly intervals.

\section{STATISTICAL ANALYSIS}

All averaged results were expressed as mean (SEM). Spectral and non-spectral measures of heart rate variability in the two groups were compared with the Mann-Whitney U test. Analyses of circadian rhythm in heart rate variability were carried out with repeated measures analysis of variance of the variables: $\log$ high frequency amplitude, log low frequency amplitude, and log of the ratio of low to high frequency. Two sided $p$ values were considered significant at the $5 \%$ level.

The study was approved by the Newham Health District Ethics Committee, and all patients gave informed consent.

\section{Results}

PATIENTS

Forty-four patients with stable angina (group A) and 131 patients in the early postinfarction period (group B) were studied. The two groups were similar in age $(61.3(1.4) v$ $62 \cdot 0(0 \cdot 9)$ and sex $(80 \% v 84 \%$ men $)$.

\section{ISCHAEMIC EPISODES}

Analysis of the 48 hour Holter recordings identified 337 ischaemic episodes in 35 patients in group A, and 370 ischaemic episodes in 65 patients in group B. In group A, 12 patients had only silent episodes, four had only painful episodes, and 19 had both. By contrast, 63 patients in group B had only silent ischaemia and the remaining two had both silent and painful episodes $\left(\chi^{2}=47 \cdot 7, p\right.$ $<0.0001)$. Figure 1 shows the number and 
Table Heart rate variability (mean (interquartile range))

\begin{tabular}{lccl}
\hline & $\begin{array}{c}\text { Group } A \\
(n=24)\end{array}$ & $\begin{array}{l}\text { Group } B \\
(n=36)\end{array}$ & $p$ Value \\
\hline High frequency peak (ms) & $10 \cdot 7(7-14)$ & $6 \cdot 7(4-9)$ & $0 \cdot 0007$ \\
Low frequency peak (ms) & $23 \cdot 7(16-28)$ & $13 \cdot 0(8-15)$ & $0 \cdot 0001$ \\
SDANN (ms) & $130(106-144)$ & $66(53-78)$ & $0 \cdot 0001$ \\
SD (ms) & $53(41-60)$ & $33(23-40)$ & $0 \cdot 0001$ \\
rMSSD (ms) & $25(17-28)$ & $20(14-25)$ & $0 \cdot 02$ \\
pNN50 (\%) & $5 \cdot 5(1 \cdot 7-6 \cdot 5)$ & $3 \cdot 4(0 \cdot 4-5 \cdot 3)$ & $0 \cdot 02$ \\
SDRR (ms) & $143(122-156)$ & $78(60-86)$ & 0.0001 \\
\hline
\end{tabular}

pNN50, proportion of adjacent RR intervals more than $50 \mathrm{~ms}$ different; $r M S S D$, root mean square of difference of successive $R R$ intervals; $S D$, mean of all 5 min standard deviations of $R R$ square of difference of successive RR intervals; $S D$, mean of all 5 min standard deviations of RR intervals; SDANN,

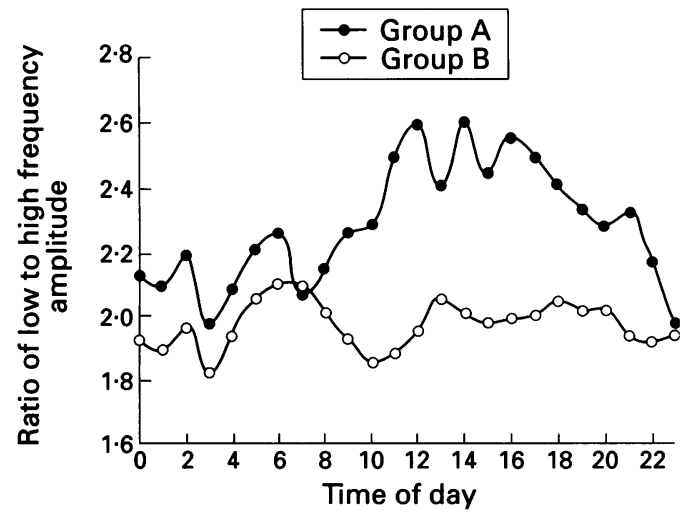

Figure 3 Circadian variation in ratio of low to high frequency spectral measures of heart rate variability as a marker of sympathovagal balance over 24 one hour periods in patients with stable angina (group $A$ ) and after myocardial infarction (group B). episodes $(65 \%)$ occurred from 0600 to 1800 compared with 119 episodes (35\%) during the right $(\mathrm{p}<0.001$, Wilcoxon signed rank test). In group $B$, however, this rhythm was abolished, with a tendency to more episodes during the night. Thus there were 157 episodes $(42 \%)$ during the day and 213 episodes $(58 \%)$ during the night $(p=0.08)$.

\section{HEART RATE VARIABILITY}

Analysis of the first 24 hours of each Holter recording showed that spectral and non-spectral measures of heart rate variability were higher in group A than group B (table). In the group as a whole, the high frequency spectral component of heart rate variability showed a clear circadian rhythm $(p=0.0001)$. In group A there was a peak at night $(1800-0600)$ of $11.1 \mathrm{~ms}$ and a daytime $(0600-1800)$ trough of $9.1 \mathrm{~ms}$. The pattern in group B was not significantly different $(p=0 \cdot 12)$, but seemed slightly less with a peak of $7 \cdot 2 \mathrm{~ms}$ and a

Figure 2 Circadian variation in low and high frequency spectral measures of heart rate variability over 24 one hour periods in patients with $(A)$ stable angina (group $A$ ) and $(B)$ after myocardial infarction (group B).

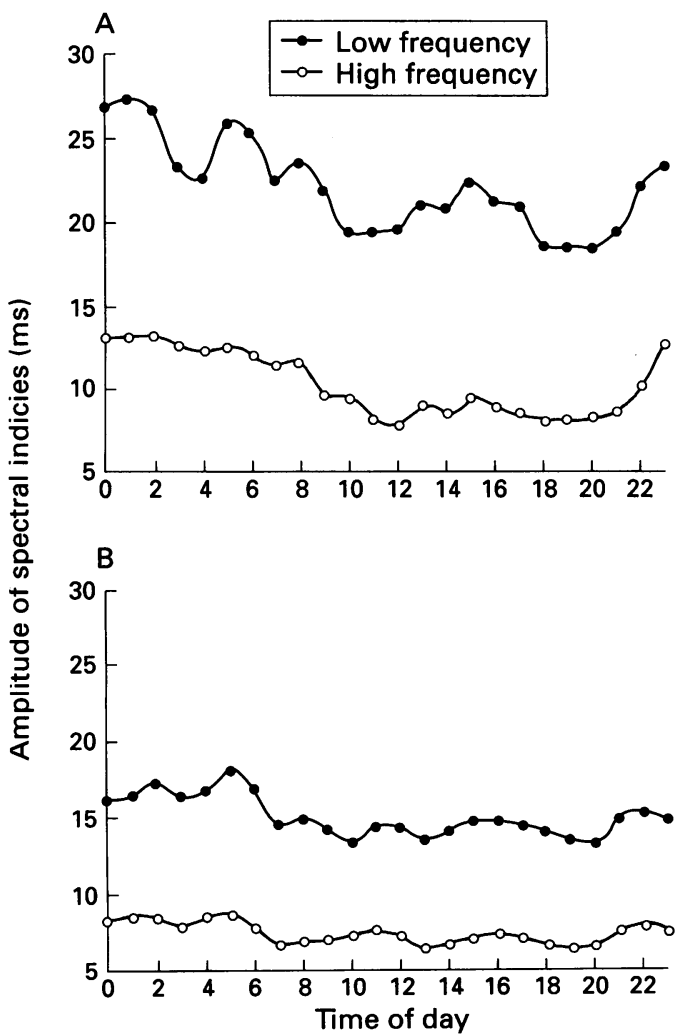

trough of $6 \cdot 6 \mathrm{~ms}$. A similar circadian variation was seen in the low frequency range $(p=$ 0.0001 ), which, although it seemed less pronounced in group B, was not significantly different between the groups $(p=0.72)$. Group A had a daytime peak of $22.7 \mathrm{~ms}$ and a nighttime trough of $21.4 \mathrm{~ms}$; in group $B$ the amplitudes were 13.5 and $12.6 \mathrm{~ms}$ (fig 2 ).

\section{SYMPATHOVAGAL BALANCE}

When the ratio of low to high frequency variability was analysed to provide a measure of sympathovagal balance, the circadian rhythm changed. In the patients as a whole, there was a significant circadian variation $(p<0.002)$ that differed between the groups $(p=0.06)$. Figure 3 shows that in group A, peak values occurred during the day with a trough at night $(2.39 v 2 \cdot 18)$, but this was clearly abolished in group B (1.98 $v 1.95)$.

\section{Discussion}

Mechanisms of ambulatory myocardial ischaemia remain uncertain. Ischaemia seems to represent a complex imbalance of oxygen supply and demand provoked partly by increases in heart rate ${ }^{16}$ and blood pressure, ${ }^{17} 18$ and partly by increases in coronary vascular tone. ${ }^{18}$ Any mechanistic theory of ambulatory ischaemia must be able to account for its circadian rhythm, which has been shown in previous studies of patients with stable angina ${ }^{1}$ and confirmed in our present study. Deedwania and Nelson showed that the peak of ischaemic episodes in the second quarter of the day was associated with a simultaneous peak of heart rate and blood pressure and as the minute by minute control of these haemodynamic variables is predominantly a function of the autonomic nervous system, they suggested that surges in sympathetic activity at this time of day might be responsible for their findings. ${ }^{19}$

Our present study, in which Holter monitoring permitted simultaneous analysis of ST segment changes and heart rate variability, provides further evidence implicating the autonomic nervous system as an important determinant of the circadian rhythm of 
ambulatory myocardial ischaemia. Pagani et al have suggested that spectral analysis of heart rate variability can provide information on the interaction between sympathetic and parasympathetic regulatory activities, with the low frequency component reflecting the level of sympathetic drive to the heart and the high frequency component (which disappears after a dose of of atropine) reflecting vagal activity. ${ }^{20}$ Thus the low to high frequency ratio provides a convenient index of sympathovagal balance. ${ }^{21}$ We have applied spectral analysis of heart rate variability to the patients in this study and have shown that in stable angina, a circadian rhythm of sympathovagal balance exists, peaking during the day at the time when ambulatory myocardial ischaemia is also at its peak. Early after myocardial infarction, on the other hand, when autonomic function is known to be deranged, the circadian rhythm of sympathovagal balance was severely lessened due largely to loss of the morning sympathetic surge. Perhaps because of this, the morning peak of ambulatory ischaemia was also lost. Of course, early after a myocardial infarction treated by thrombolysis, plaque instability and intracoronary thrombotic debris can cause ongoing ischaemia, ${ }^{22}$ independently of autonomic influences, and this might obscure any relation that exists. Nevertheless, the data provide circumstantial evidence supporting the hypothesis of Deedwania and Nelson that in patients with stable angina the circadian rhythms of ambulatory ischaemia and sympathovagal activity may be causally inter-related. ${ }^{19}$

The hypothesis that the circadian rhythm of ambulatory ischaemia in patients with stable angina is caused by fluctuations in sympathetic activity is attractive for three reasons. It is consistent with the known circadian rhythms of blood catecholamine ${ }^{23}$ and corti$\mathrm{sol}^{24}$ concentrations that also peak in the second quarter of the day. It is also consistent with reports that $\beta$ blockers are effective in reducing the frequency and the circadian variation of ambulatory ischaemia. ${ }^{25}$ Finally, it is consistent with current concepts of the pathophysiology of ambulatory myocardial ischaemia, which view it as the combined response to increases in oxygen demand and reductions in oxygen supply, in a variable ratio. ${ }^{26}$ Thus sympathetically driven increments in heart rate and blood pressure would be expected to provoke ischaemia through parallel increases in myocardial oxygen demand. Patients with stable angina, however, are often predisposed to exaggerated vasoconstriction due to the development of endothelial dysfunction in relation to atherosclerotic plaques, ${ }^{27}$ and when this is the predominant response to a sympathetic stimulus, it can lead to supply driven ischaemia without a large increase in myocardial oxygen demand. This is regarded as the most likely mechanism for those episodes of ambulatory ischaemia that occur without a preceding increase in heart rate or blood pressure. ${ }^{26}$

The circadian rhythm of sympathovagal activity in patients with stable coronary artery disease may also account for previously described circadian rhythms of out of hospital sudden cardiac deaths ${ }^{2}$ and onset of acute myocardial infarction, ${ }^{5}$ both of which peak in the second quarter of the day. Thus it is possible to speculate that the same sympathetic drive that provokes the morning peak in myocardial ischaemic episodes might also provoke cardiac arrhythmias or plaque events, either by direct effects on the myocardium or indirectly through provocation of ischaemia and surges in blood pressure. This may have important implications for prophylactic treatment if effective suppression of the morning peak in sympathetic drive could be achieved by giving $\beta$ blockers.

In conclusion, this study has shown that in stable angina the circadian rhythm of myocardial ischaemia, peaking during the day time hours and dipping at night, is associated with a similar circadian rhythm of sympathovagal balance. In the early postinfarction period, both the ischaemic and sympathovagal rhythms are severely diminished or lost altogether. Although causality cannot be proved, the data suggest that circadian changes in sympathovagal tone may explain, at least in part, the circadian rhythm of ambulatory myocardial ischaemia in patients with stable angina.

BM is funded by the British Heart Foundation.

1 Mulcahy D, Keegan J, Crean P, Quyyumi A, Shapiro L, Wright C, et al. Silent myocardial ischaemia in chronic stable angina: a study of its frequency and characteristics in 150 patients. Br Heart $\mathcal{F} 1988 ; 60: 417-23$

2 Willich SN, Levy D, Rocco MB, Tofler GH, Stone PH, Muller JE. Circadian variation in the incidence of sudden cardiac death in the Framingham heart study population. Am F Cardiol 1987;60:801-6.

3 Behar S, Reicher-Reiss H, Goldbourt U, Kaplinsky E. Circadian variation in pain onset in unstable angina pectoris. Am f Cardiol 1991;67:91-3.

4 Thompson DR, Sutton TW, Jowett NI, Pohl JE. Circadian variation in the frequency of onset of chest pain in acute myocardial infarction. Br Heart $f$ 1991; 65:177-8.

5 Muller JE, Stone PH, Turi ZG, Rutherford JD, Czeisler $\mathrm{CA}$, Parker C, et al. Circadian variation in the frequency $\mathrm{CA}$, Parker C, et al. Circadian variation in the frequency
of onset of acute myocardial infarction. $N$ Engl $\mathcal{F}$ Med

6 Tofler GH, Brezinski D, Schafer AI, Czeisler CA, Rutherford JD, Willich SN, et al. Concurrent morning increase in platelet aggregability and the risk of myocardial infarction and sudden cardiac death. $N$ Engl $7 \mathrm{Med}$ 1987;316:1514-8.

7 Rosing DR, Brakman P, Redwood DR, Goldstein RE, Beiser GD, Astrup T, et al. Blood fibrinolytic activity in man: diurnal variation and the response to varying intensities of exercise. Circ Res 1970;28:171-83.

8 Andreotti F, Davies GJ, Hackett DR, Khan MI, De Bart AC, Aber VR, et al. Major circadian fluctuations in fibrinolytic factors and possible relevance to time of onset of myocardial infarction, sudden cardiac death and stroke. Am $f$ Cardiol 1988;62:635-7.

9 Willich SN, Lowel H, Lewis M, Arntz R, Baur R, Winther $\mathrm{K}$, et al. Association of wake time and the onset of myocardial infarction. Triggers and mechanisms of myocardial infarction (TRIMM) pilot study. TRIMM myocardial infarction (TRIMM) pilot study. TR.

10 Mulcahy D, Purcell H, Fox K. Should we get up in the morning? Observations on circadian variations in cardiac morning? Observations on circadian

11 Taylor CR, Hodge EM, White DA. Circadian rhythm of . angina: similarity to circadian rhythms of myocardia infarction, ischemic ST segment depression, and sudden
cardiac death. The Amlodipine Angina Study Group. cardiac death. The Amlodipi

12 Mickley H, Pless P, Nielsen JR, Moller M. Circadian variation of transient myocardial ischemia in the early outation of transient myocardial ischemia in the early out-
of-hospital period after first acute myocardial infarction. of-hospital period after first acure

13 Fox JP, Beattie JM, Salih MM, Davies MK, Littler WA, Murray RG. Silent ischaemia following myocardial infarction: frequency, characteristics and prognosis. Eur Heart $\mathcal{f}$ 1988;9(suppl N):108-13. 
14 Moczurad KW, Grodecki JK, Dubiel JP, Curylo AM. Silent myocardial ischaemia in Holter monitoring and exercise stress testing after a first myocardial infarction. Eur Heart f 1988;9(suppl N):114-8.

15 Casolo GC, Stroder P, Signorini C, Calzolari F, Zucchini $\mathrm{M}$, Balli $\mathrm{E}$, et al. Heart rate variability during the acute phase of myocardial infarction. Circulation 1992;85: 2073-9.

16 Quyyumi AA, Wright CA, Mockus LJ, Fox KM Mechanisms of nocturnal angina pectoris: importance of increased myocardial oxygen demand in patients with severe coronary artery disease. Lancet 1984;1: 1207-9.

17 Rozanski A, Bairey CN, Krantz DS, Friedman J, Resser $\mathrm{KJ}$, Morell $\mathrm{M}$, et al. Mental stress and the induction of silent myocardial ischemia in patients with coronary artery disease. N Engl F Med 1988;318:1005-12.

18 Deanfield JE, Shea $M$, Kensett $M$, Horlock P, Wilson RA, de-Landsheere CM, et al. Silent myocardial ischaemia de-Landsheere CM, et al. Silent myocardial
due to mental stress. Lancet 1984;2:1001-5.

19 Deedwania PC, Nelson JR. Pathophysiology of silent myocardial ischemia during daily life. Hemodynamic evaluation by simultaneous electrocardiographic and blood pressure monitoring. Circulation 1990;82:

20 Pagani M, Mazzuero G, Ferrari A, Liberati D, Cerutti S, Vaitl $\mathrm{D}$, et al. Sympathovagal interaction during mental stress. A study using spectral analysis of heart rate vari- ability in healthy control subjects and patients with a ability in healthy control subjects and patients with a
prior myocardial infarction. Circulation 1991;83(suppl prior my
II):43-51

21 Lombardi F, Sandrone G, Pernpruner S, Sala R, Garimoldi M, Cerutti S, et al. Heart rate variability as an index of sympathovagal interaction after acute myocardial infarction. Am $\mathcal{F}$ Cardiol 1987;60:1239-45.

22 Davies SW, Marchant B, Lyons JP, Timmis AD, Rothman $\mathrm{MT}$, Layton CA, et al. Irregularity of coronary lesion after thrombolysis predicts early clinical instability. $7 \mathrm{Am}$ Coll Cardiol 1991;18:669-74.

23 Turton MB, Deegan T. Circadian variations of plasma catecholamine, cortisol, and immunoreactive insulin concentrations in supine subjects. Clin Chim Acta 1974 55:389-97.

24 Weitzman ED, Fukushima D, Nogeire C, Roffwarg $\mathrm{H}$, Gallagher TH, Hellman L. Twenty-four hour pattern of the episodic secretion of cortisol in normal subjects. f Clin Endocrinol Metab 1971;33:14-22.

25 Goldstein S. Effect of beta-adrenergic blocking agents on the circadian occurrence of ischemic cardiovascular the circadian occurrence of ischemic

26 Stone PH. Unraveling the mechanisms of ambulatory ischemia: how and why. Circulation 1990;82 1528-30

27 Harrison DG, Freiman ML, Marcus ML, Heistad DD. Alterations of vascular reactivity in atherosclerosis. Circ Res 1987;61 (suppl II): 74-80. 